\title{
Über den Einfluß des Wachstumszustandes eines Bakteriums auf seine Strahlenempfindlichkeit
}

\author{
Von Thea Houtermans \\ Aus dem Hygiene-Institut der Universität Göttingen (Direktor: Prof. Dr. F. S ch ütz) \\ (Z. Naturforschg. 8 b, 767-771 [1953]; eingegangen am 12. August 1953)
}

\begin{abstract}
An relativ gleichmäßig auf Agar auskeimenden Kulturen von E. coli wird die Abhängigkeit der UV- und $\alpha$-Strahlen-Empfindlichkeit vom Wachstumszustand der Einzelindividuen untersucht. Der Übergang aus einer ruhenden Bouillonkultur in den Zustand des Wachsens auf Agar bewirkt eine starke Erhöhung der UV-Sensibilität, während die Empfindlichkeit gegenüber $\alpha$-Strahlen hierbei nur wenig oder nicht beeinflußt wird. Im weiteren Verlauf des Wachstums zeigt sich, daß die Bakterien sowohl gegen UV- wie gegen $\alpha$-Strahlen kurz nach der Teilung am empfindlichsten, kurz vor der Teilung am unempfindlichsten sind.
\end{abstract}

Es: ist verschiedentlich gezeigt worden, daß bei der Strahleninaktivierung von Bakterien der Effekt durch zahlreiche Faktoren beeinflußt werden kann. Je nach den Bedingungen vor der Bestrahlung (aerobes oder anaerobes Wachstum der Kultur ${ }^{1}$, „Wärmesensibilisierung "2), während der Bestrahlung (Sauerstoffgehalt des Milieus ${ }^{1}$, Temperatur ${ }^{3}$ ) oder nach der Bestrahlung (Bebrütungstemperatur ${ }^{4}$, Nährbodenzusammensetzung ${ }^{4}$, zusätzliche Bestrahlung mit sichtbarem Licht ${ }^{5}$ ) können verschiedene Inaktivierungsprozentsätze resultieren. Es ist von vornherein wahrscheinlich, daß auch der Wachstumszustand eines Bakteriums, der etwa durch die seit der letzten Teilung verflossene Zeit charakterisiert werden kann, die Strahlenempfindlichkeit beeinflußt.

Für derartige Untersuchungen müßten jedoch Kulturen zur Verfügung stehen, bei denen sich alle Bakterien in einem annähernd gleichen Wachstumszustand befinden, also sich etwa zur gleichen Zeit teilen. Wenn man die üblicherweise verwendeten Bouillonkulturen auf Agar wachsen läßt, so ist dies sicher nicht der Fall. Im Rahmen einer anderen Problemstellung war es jedoch gelungen, sehr gleichmäßig auf Agar auskeimende Kulturen von E.coli (HIG) zu erzielen ${ }^{6}$. Es lag nun nahe, zu untersuchen, ob sich mit diesem Bakterienmaterial eine Abhängigkeit der Strahlenempfindlichkeit vom Wachstumszustand nachweisen läßt.

1 A. Holla ender, G. E.Stapleton u. F. L. Martin, Nature [London] 167, 103 [1951].

2 H. Langendorff u. K. S o mmermeyer, Z. Naturforschg. 8 b, 118 [1953].

3 R. B. Jr. Mefford u. L. B. Campbell, Proc. Soc. exp. Biol. Med. 79, 12 [1952].

4 W. H a rm u. W.Stein. Z. Naturforschg. 8 b, 123 [1953].

\section{Wachstum der Versuchsbakterien}

Züchtung der Ausgangskultur: Ausgehend von eineı Stichagarkultur von E.coli (HIG) wurde nach mehreren Passagen über Fleischwasserbouillon eine Fleischwasserbouillonagarplatte dünn beimpft und eine einzelne Kolonie wieder in Bouillon übertragen. Nach 14-18-stdg. Bebrütung bei $37^{\circ} \mathrm{C}$ wurde eine Öse in Fleischwasserbouillon mit Zucker geimpft und etwa $7 \mathrm{Stdn}$. bebrütet. Diese Kultur, die sich am Anfang der maximalen stationären Wachstumsphase befand, wurde für die Versuche verwendet.

Wenn man nun diese Bakterien auf Agar überträgt, bei $37^{\circ}$ bebrütet und nach verschiedenen Zeiten Kontrollen unter dem Mikroskop untersucht, so hat man den Eindruck eines recht gleichmäßigen Wachstums. Im Idealfall müßten sich natürlich alle Bakterien immer zum gleichen Zeitpunkt teilen. Die Wachstumskurve, die hier als die mittlere Anzahl von Bakterien pro Kolonie in Abhängigkeit von der Bebrütungszeit definiert werden soll (auch ein einzeln liegendes Bakterium ist in diesem Sinn eine Kolonie), würde also eine Treppenfunktion sein, wie sie in Abb. 1 dargestellt ist. Die Generationsdauer, also hier der Abstand zwischen den einzelnen Stufen, nimmt beobachtungsgemäß im Anfang des Wachstums bis zum Eintritt in die logarithmische Phase ab. Tatsächlich sind die Stufen der Wachstumskurve weitgehend verwischt, jedoch sind sie z. B. bei der in Abb. 1 eingezeichneten experimentellen Kurve in der Nähe von 2, 4 und 8 Bakterien im Mittel pro. Kolonie noch deutlich erkennbar. Die Steigung der Kurve ist ein Maß für den Prozentsatz von Bakterien, die jeweils in der Teilung begriffen sind.

5 A. Kelner, J. Bacteriol. 58, 511 [1949].

6 T. Houtermans, Strahlentherapie, im Druck. 


\begin{tabular}{|c|c|c|c|c|c|c|c|c|c|c|}
\hline \multirow{2}{*}{$\begin{array}{l}\text { Bebrü- } \\
\text { tungszeit } \\
\text { min }\end{array}$} & \multicolumn{9}{|c|}{$\begin{array}{l}\text { Prozentsatz } \\
\text { der Kolonien mit } n \text { Bakterien }\end{array}$} & \multirow{2}{*}{$n$} \\
\hline & $n=1$ & 2 & 3 & 4 & 5 & 6 & 7 & 8 & 9 & \\
\hline 0 & 96 & 4 & - & - & - & - & - & - & - & 1,04 \\
\hline 18 & 95 & 5 & - & - & - & - & - & - & - & 1,05 \\
\hline 30 & 80 & 20 & - & - & - & - & - & - & - & 1,20 \\
\hline 45 & 47 & 53 & - & - & - & - & - & - & - & 1,53 \\
\hline 60 & 35 & 65 & - & - & - & - & - & - & - & 1,65 \\
\hline 65 & 31 & 68 & - & 1 & - & - & - & - & - & 1,69 \\
\hline 71 & 23 & 75 & 1 & 1 & - & - & - & - & - & 1,75 \\
\hline 77 & 22 & 76 & 1 & 1 & - & - & - & - & - & 1,77 \\
\hline 83 & 19 & 77 & 1 & 3 & - & - & - & - & - & 1,88 \\
\hline 89 & 10 & 79 & 5 & 6 & - & - & - & - & - & 2,07 \\
\hline 96 & 10 & 36 & 9 & 45 & - & - & - & - & - & 2,85 \\
\hline 103 & 11 & 14 & 11 & 62 & 1 & 1 & - & - & - & 3,3 \\
\hline 109 & 12 & 8 & 7 & 66 & 5 & - & 2 & - & - & 3,5 \\
\hline 115 & 8 & 7 & 7 & 61 & 6 & 6 & 4 & 1 & - & 3,9 \\
\hline 121 & 9 & 2 & 4 & 52 & 13 & 6 & 7 & 7 & - & 4,3 \\
\hline 127 & 6 & 2 & 4 & 23 & 6 & 19 & 13 & 26 & 1 & 5,7 \\
\hline 135 & 4 & 2 & 2 & 8 & 8 & 17 & 28 & 29 & 2 & 6,4 \\
\hline
\end{tabular}

Tab. 1. Prozentsätze der Kolonien mit $n$ Bakterien in Abhängigkeit von der Bebrütungszeit.

In Tab. 1 sind die zu der Kurve in Abb. 1 gehörigen Prozentsätze der Kolonien mit 1, 2, 3 usw. Bakterien pro Kolonie in Abhängigkeit von der Bebrütungszeit angegeben, aus denen die Berechnung der Mittelwerte erfolgte. Hier erkennt man noch klarer als aus den Mittelwerten das relativ gleichmäßige Wachstum.

Auch bei Verwendung der wie beschrieben gezüchteten Kulturen waren die Kurven oft nicht befriedigend (z. B. Abb. 5). Ferner waren gelegentlich auch zusätzliche Stufen bei etwa 3 und etwa 6 Bakterien im Mittel pro Kolonie zu erkennen. Dies könnte auf 2 Varianten hinsichtlich der Wachstumsgeschwindigkeit hinweisen.

\section{Versuchsmethodik}

Um die Abhängigkeit der Strahlenempfindlichkeit von dem Wachstumszustand zu prüfen, wurde der Kolonienprozentsatz bei konstanter Bestrahlungsdosis mit UV- und $\alpha$-Strahlen als Funktion der Vorbebrïtungszeit auf Agar ermittelt.

Aus einer $2 \mathrm{~mm}$ dicken Agarplatte wurden kreisrunde Plättchen mit $1 \mathrm{~cm}$ Durchmesser ausgestochen und auf Objektträger gelegt. Aus der geeignet verdünnten Bakterienemulsion wurde dann auf jedes Plättchen aus einer Kapillare ein Tropfen von etwa $3 \mathrm{mg}$ geblasen, dessen Volumen im Rahmen der Meßgenauigkeit reproduzierbar war. Ein Plättchen wurde sofort nach Eintrocknen des Tropfens bestrahlt, alle anderen in den Brutschrank in eine dort schon vorgewärmte feuchte Kammer gesetzt. Nach der jeweils gewünschten Bebrütungszeit wurden immer zwei Plättchen herausgenommen, von denen eines sofort bestrahlt, das andere dagegen in eine feuchte Kammer mit Formalin gesetzt wurde, so daß das Wachstum momentan angehalten war. Diese Plättchen wurden für die Ermittlung der Wachstumskurven verwendet.

Die bestrahlten Plättchen wurden in Reagensgläser mit physiologischer Kochsalzlösung gebracht und gut geschüttelt. Die Menge der zur Abschwemmung benutzten Kochsalzlösung wurde so gewählt, daß bei den vermuteten jeweiligen Inaktivierungsprozentsätzen zwischen 300 und 600 Kolonien auf einer Platte zu erwarten waren. Tatsächlich lagen die Kolonienzahlen pro Platte zwischen 200 und 1200. Vorversuche hatten ergeben, daß durch dieses Verfahren die Bakterien ausreichend quantitativ abgeschwemmt wurden. Anschließend wurde $0,1 \mathrm{~cm}^{3}$ der Lösung auf Fleischwasserbouillonagarplatten pipettiert und diese sofort in einen Brutschrank mit $37^{\circ}$ gesetzt.

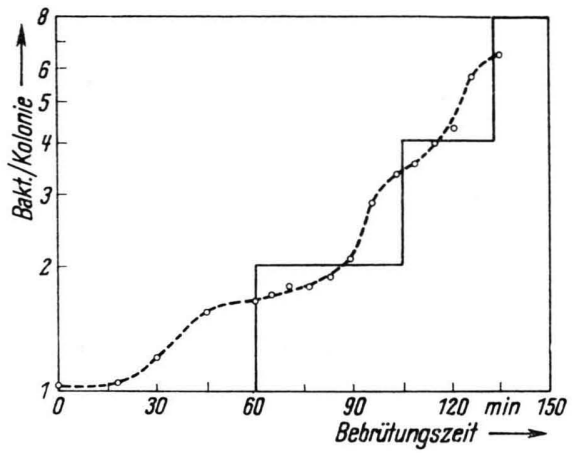

Abb. 1. Theoretische Wachstumskurve für vollkommen gleichmäßiges Wachstum und experimentelle Kurve.

Die Auszählung der Kolonien erfolgte nach 24 Stdn. mit bloßem Auge.

Zur Berechnung der Kolonienprozentsätze brauchte eine Nullplatte nur von den unbebrüteten Bakterien angelegt zu werden. Die Gesamtzahl der auf eine Platte übertragenen vorbebrüteten Bakterien wurde durch Multiplikation der Kolonienzahl auf der Nullplatte mit dem sich aus der Wachstumskurve ergebenden Mittelwert der Anzahl Bakterien pro Kolonie berechnet.

Trotz noch so kräftigen Schüttelns ließ sich keine völlige Vereinzelung der auf dem Agar im Wachstum begriffenen Bakterien erreichen. Eine gewisse Anzahl lag stets in Paaren vor; Klumpen von 3 und mehr Bakterien traten jedoch schon nach relativ leichtem Schütteln nicht mehr auf. Der Prozentsatz der Paare nimmt natürlich zunächst mit wachsender Vorbebrütungszeit zu und bleibt dann ziemlich konstant. Nachdem sich die meisten Bakterien einmal geteilt hatten, lagen die ermittelten Werte stets zwischen 35 und 25\%. Die Korrektur, die sich hieraus für die Prozentsätze der aktiven Keime ergibt, ist jedoch nur klein, da bei der obigen Berechnung der durch die Doppelten bedingte Fehler nur in die Anzahl der bestrahlten aktiven, nicht aber in die Gesamtzahl der auf die Platte geimpften Keime eingeht.

Ist $N$ der experimentell ermittelte Prozentsatz der aktiven Keime, so ist der tatsächliche, korrigierte Prozentsatz $N^{*}$, wie eine einfache Rechnung ergibt, 
$N^{*}=N \cdot f(p, N)$

$$
\text { wobei } f(p, N)=\frac{\left(1-\sqrt{1-\left(\begin{array}{c}
4 p N \\
(1+p)^{2}
\end{array}\right)(1+p)^{2}}\right.}{2 p N}
$$

$(p=$ Prozentsatz der Paare).

In Abb. 2 ist eine Kurvenschar von $f(p, N)$ in Abhängigkeit von $N$ mit $p$ als Parameter gegeben.

Die Wachstumskurve wurde im Phasenkontrastmikroskop mit 90-facher Objektiv- und 12-facher Okularvergrößerung ausgezählt. Die Teilung eines Bakteriums

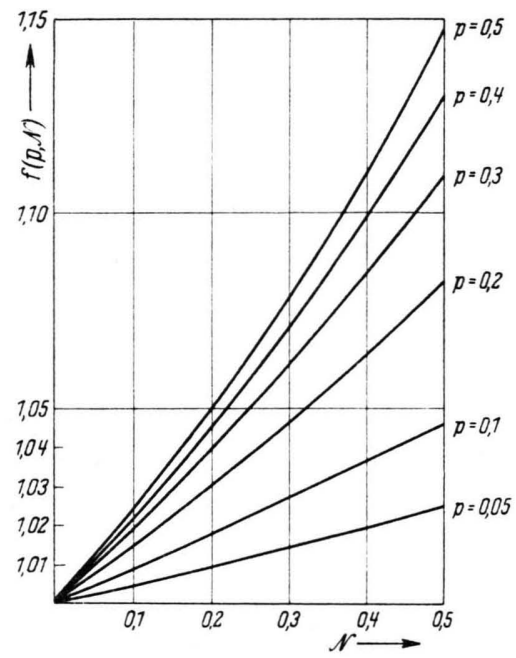

Abb. 2. Kurvenschar für den Korrekturfaktor $f(p, N)$.

wurde als vollzogen angesehen, wenn eine Abschnürung deutlich war. Vor der Zählung mußte auf das Agarplättchen ein Deckglas gelegt werden. Eine Verwischung der Kolonien ist bei einiger Vorsicht leicht zu vermeiden.

Zur Ermittlung der Kolonienprozentsätze war das früher häufig angewandte mikroskopische Zählverfahren nicht geeignet, da die im Wachstum begriffenen Bakterien nach der Bestrahlung wesentlich stärker zur Bildung von Riesenformen neigten als Bakterien aus der stationären maximalen Phase. Vor allem wuchsen auch die inaktivierten Keime zunächst zu kleinen „Schlangenkolonien“ heran, über deren weitere Entwicklung nur Mutmaßungen möglich erschienen.

Als Strahlenarten wurden UV- und $\alpha$-Strahlen verwandt. Als UV-Quelle diente das Gesamtspektrum einer Quecksilberhochdrucklampe der Firma Heraeus, Hanau, vom Typ S 81, die bei einer Stromstärke von 1,4 A betrieben wurde und $50 \mathrm{~cm}$ vom Objekt entfernt war. Die Bestrahlungszeit betrug 10 sec. Als $\alpha$-Strahlen-Quelle wurde ein Thorium-B-Präparat verwandt (Meßmethode und Eichung siehe 1.c. ${ }^{7}$ ). Die Dosis lag zwischen 100 und $225 \alpha$-Teilchen pro $\mu^{2}$, die Bestrahlungszeit zwischen 75 und $100 \mathrm{sec}$. Die Dosisleistung nahm während der Versuchsdauer von etwa 2,5 Stdn. entsprechend der Halb-

7 H. Engelhard u. T. Houtermans, Z. Naturforschg. 5 b, 267 [1950]. wertszeit des Th B von etwa 10,6 Stdn. ab. Durch entsprechende Verlängerung der Bestrahlungszeiten wurde jedoch die Dosis innerhalb einer Versuchsreihe konstant gehalten.

\section{Versuchsergebnisse und Diskussion}

In den Abb. 3, 4 und 5 sind einige Versuchsergebnisse zusammengestellt. Bei den Versuchen 4 und 5 sind die UV- und $\alpha$-Bestrahlungen an Bakterienmaterial derselben Ausgangskultur gleichzeitig durchgeführt. Die Wachstumskurven in Abb. 3 und 4 entsprechen etwa den Forderungen, die oben für relativ

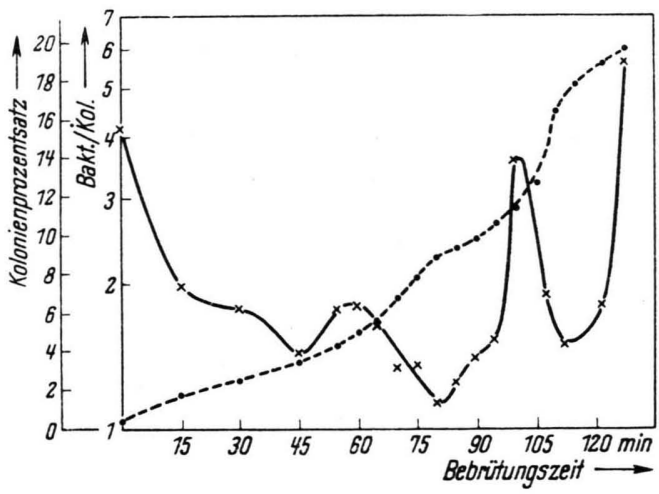

Abb. 3. Prozentsatz aktiver Keime nach UV-Bestrahlung $(\cdot--\cdot)$ und Wachstumskurve $(\times-\times)$ als Funktion der Vorbebrütungszeit auf Agar.

gleichmäßiges Wachstum gestellt wurden; jeweils bei 2, 4 und 8 Bakterien im Mittel pro Kolonie sind Stufen angedeutet. (Die Wachstumskurven der Abb. 1 und 4 sind identisch.) In Abb. 5 dagegen ist dies nicht genügend deutlich der Fall.

Aus Abb. 3 folgt für UV-Strahlung:

1. Der Úbergang eines Bakteriums aus einer Bouillonkultur, die sich in der maximalen stationären Phase befindet, in den Zustand des Wachstums auf Agar bedingt eine Erhöhung der Strahlenempfindlichkeit. Die mikroskopische Beobachtung zeigt, daß die Bakterien dabei nur langsam etwas größer und kontrastreicher werden.

2. Beim Wachsen auf Agar ist ein Bakterium am strahlenempfindlichsten kurz nach und am unempfindlichsten kurz vor der Teilung.

Abb. 4 bestätigt diese Zusammenhänge. Die Steigerung der UV-Sensibilität nach kurzer Vorbebrütung ist hier noch erheblich größer. Vor der ersten Teilung ist kein Maximum erkennbar; dieses war auch bei den hier nicht wiedergegebenen Versuchsreihen 
verschieden deutlich. Eine Erklärung hierfür wäre eine unterschiedliche Überlagerung der Effekte 1 u. 2, je nach der Ausgangskultur.

Bei dem Versuch der Abb. 5 ist, da die Wachstumskurve nicht die gewünschte Form zeigt, nicht pro Generationsdauer ein Maximum zu erwarten. Auch

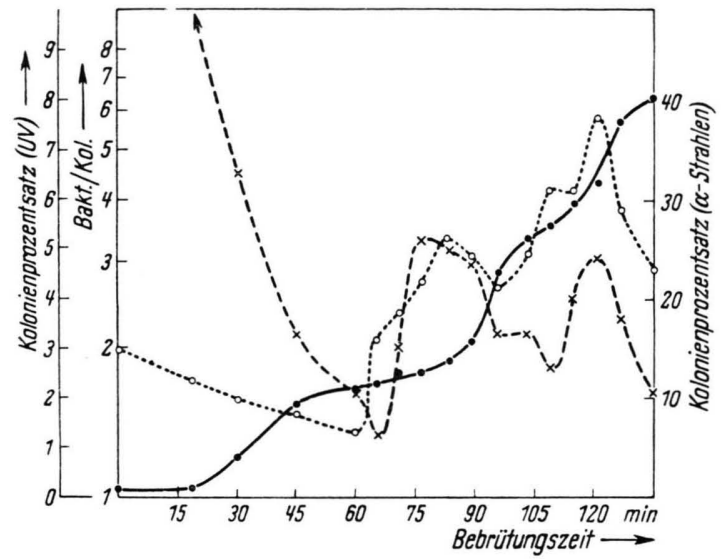

Abb. 4. Prozentsatz aktiver Keime nach UV-Bestrahlung $(\times--\times)$ und $\alpha$-Bestrahlung $(0 \ldots \ldots)$ ) und Wachstumskurve $(\cdot-\cdot)$ als Funktion der Vorbebrütungszeit auf Agar.

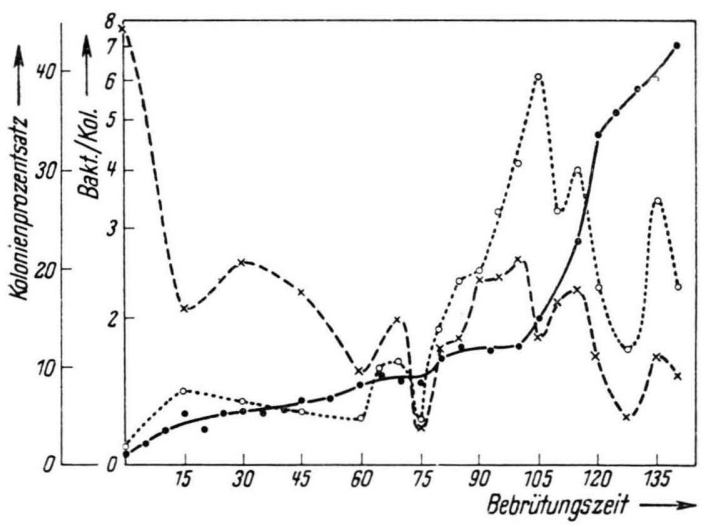

Abb. 5. Prozentsatz aktiver Keime nach UV-Bestrahlung $(x-\cdots \times)$ und $\alpha$-Bestrahlung $(0, \ldots, 0)$ und Wachstumskurve (.- - )als Funktion der Vorbebrütungszeit auf Agar.

hier ist jedoch im Prinzip die Zuordnung der Steigung der Wachstumskurve zu dem Prozentsatz der aktiven Keime gewahrt.

Ein Vergleich der Inaktivierungskurven für $\alpha$ Strahlen in Abb. 4 und 5 mit den zugehörigen UVKurven zeigt, daß die mit demselben Bakterienmaterial ausgeführten Versuche für höhere Vorbebrütungszeiten einander entsprechen; Maxima und Minima liegen etwa an den gleichen Stellen. Auch gegenüber $\alpha$-Strahlen ist also die Empfindlichkeit der Bak- terien kurz nach der Teilung am größten, kurz vor der Teilung am kleinsten. Die Änderung der Strahlenempfindlichkeit beim Übergang vom Ruhezustand in Bouillon zum Wachstum auf Agar ist hier jedoch nicht erheblich und in den einzelnen Versuchen verschieden. Bei manchen Versuchen blieb auch der Kolonienprozentsatz im Anfang der Vorbebrütung konstant.

Die Kurvenmaxima kurz vor der Teilung lassen sich nicht durch die Annahme erklären, daß eine für die Strahlenresistenz maßgebliche Teilung, etwa der Gen-Substanz, bereits vollzogen ist, bevor sie bei der Zählung berücksichtigt wird. In diesem Fall würde es sich also hinsichtlich der Inaktivierung praktisch um Bakterienpaare handeln. Dies könnte jedoch allerhöchstens eine Erhöhung des Kolonienprozentsatzes um einen Faktor 2 bedingen.

Ist $W$ die Inaktivierungswahrscheinlichkeit, $(1-W)$ also die Wahrscheinlichkeit für ein Bakterium, aktiv zu bleiben, so ist die Wahrscheinlichkeit, daß ein Bakterienpaar überlebt, $\left(1-W^{2}\right)$. Der Quotient $\begin{gathered}1-W^{2} \\ 1-W\end{gathered}=1+W$ ist stets kleiner als 2. Bei den angegebenen Versuchen könnte selbst bei den höchsten Kolonienprozentsätzen dieser Effekt nur einen Faktor 1,4 ausmachen, wenn alle Bakterien praktisch doppelt wären, aber als solche nicht gezählt würden.

Die gemessenen Unterschiede zwischen den relativen Maxima und Minima der Inaktivierungskurven sind jedoch trotz der immer noch beträchtlichen Heterogenität des Versuchsmaterials erheblich größer. Die Inaktivierungswahrscheinlichkeiten der Bakterien vor und nach der Teilung müssen tatsächlich noch erheblich mehr voneinander abweichen.

Die Empfindlichkeitssteigerung der Bakterien gegenüber UV-Licht beim Übergang in den Zustand des Wachsens auf Agar entspricht den Ergebnissen, die Langendorff und Sommermeyer ${ }^{2}$ mit in Bouillon vorbebrüteten E. coli $B$ bei Röntgenbestrahlung erhielten. Langendorff und So m m e r m e y e r bezeichnen den Effekt als Wärmesensibilisierung; bei gleicher Vorbehandlung der Bakterien in NaCl-Lösung resultierte jedoch keine erhöhte Strahlenempfindlichkeit. Wahrscheinlich spielt die Wärme nur insofern eine Rolle, als sie den für die Sensibilisierung wesentlichen Übergang in den $\mathrm{Zu}$ stand des Wachsens beschleunigt, und der von den Verff. als Wärmemodifikation bezeichnete Effekt kann auch bei niedrigeren Temperaturen und entsprechend längerer Vorbebrütungszeit erzielt werden. Die Verff. machen quantitative Angaben über die Halbwertsdosen der sensibilisierten Bakterien. Die hier zitierten 
Versuche mit UV-Strahlen waren jedoch auch für geringe Vorbebrütungszeiten nur qualitativ reproduzierbar. Offenbar ist der Zustand einer 24-Stdn.-Kultur sehr viel leichter zu reproduzieren als der einer Kultur im Anfang der maximalen stationären Phase, die hier als Ausgangsmaterial benutzt wurde.

Die gemessene Abhängigkeit der $\alpha$-Strahlenempfindlichkeit vom Wachstumszustand des Bakteriums zeigt erneut (siehe auch ${ }^{6}$ ), daß der Wirkungsquerschnitt für $\alpha$-Strahlen keineswegs mit dem Querschnitt der von Piekarski als Nukleoide bezeichneten Kernäquivalente übereinstimmt, wie man aus
Messungen von Lea an ruhenden Kulturen von E. coli gefolgert hat ${ }^{8,9}$. Die starken Empfindlichkeitsänderungen während eines Generationszyklus können weder mit der Änderung der Größe noch der Anzahl der Nukleoide in Zusammenhang gebracht werden.

Diese Arbeit wurde durch die Unterstützung der D e u t schen Forschungsgemeinschaft ermöglicht.

8 D. E. Lea, „Actions of Radiations on Living Cells“, Cambridge University Press, 1946.

9 K. S ommermeyer, "Quantenphysik der Strahlenwirkung in Biologie und Medizin“, Verlagsges. Geest u. Portig, Leipzig, 1952.

\title{
Über den Einfluß des Kernes auf die Formbildung von Acetabularia in verschiedenen Entwicklungsstadien
}

\author{
Von Kurt Beth \\ Aus dem Max-Planck-Institut für Meeresbiologie, Abteilung Hä m me rling \\ (Z. Naturforschg. 8 b, 771-775 [1953]; eingegangen am 29. Juli 1953)
}

\begin{abstract}
Kernlose und kernhaltige Zellen von Acetabularia mediterranea verschiedener Entwicklungsstadien wurden vergleichend auf ihr Formbildungsvermögen untersucht. Kernlose Teile bilden desto mehr Hüte je weiter entwickelt die kernhaltigen Ausgangspflanzen sind. Kernlose Teile junger Zellen bilden früher Hüte als die kernhaltigen Kontrollpflanzen, kernlose Teile älterer Zellen gleichzeitig oder später. Die Befunde werden im Zusammenhang mit der Tatsache betrachtet, daß das starke Kernwachstum spätestens mit der Anlage des Hutes beendet ist. Auf jungen Entwicklungsstadien verhindert die Gegenwart des stark wachsenden Kernes, daß Hüte angelegt werden. Dabei werden cytoplasmatische Prozesse, deren vollständiger Ablauf zur Hutbildung erforderlich ist, blockiert. Diese können erst vonstatten gehen, wenn der Kern nicht mehr wächst oder entfernt worden ist.
\end{abstract}

1.

T $\mathrm{m}$ folgenden sollen Versuche und Beobachtungen an Acetabularia mitgeteilt werden, welche zum ersten Male auf Beziehungen zwischen der Formbildung einer Zelle und der Wachstumsphase ihres Kernes hinweisen. Frühere Versuche ${ }^{1,2}$ hatten das beträchtliche Formbildungsvermögen kernloser Zellen gezeigt, aber auch ergeben, daß die Leistungen kernloser Teile höchstens anfänglich mit denjenigen kernhaltiger Zellen Schritt halten. Nach neuen Versuchen an Acetabularia mediterranea, veranlaßt durch gleichartige Beobachtungen an A. crenulata, können kernlose Teile junger Entwicklungsstadien den gleichaltrigen kernhaltigen Zellen in der Formbildung sogar vorauseilen.

2. Entfernt man bei großen Acetabularia-Zellen, die kurz vor der Hutanlage stehen, den Kern durch

1 J. H ämmer ling, Naturwiss. 33, 337, 361 [1946]; Intern. Rev. Cytology 2 [1953].
Abschneiden des Rhizoides, so bilden trotzdem viele der kernlosen Teile Hüte, wenn auch nicht so schnell wie die kernhaltigen Pflanzen (Abb. 1a). Stellt man aber von kleineren Zellen, die so bald noch keinen Hut anlegen würden, kernlose Teile her, so entwikkeln diese früher Hüte als die kernhaltigen Kontrollpflanzen (Abb. 1c, d; Abb. 2 a, b). Der Unterschied zwischen kernlosen und kernhaltigen Zellen dieser jungen Entwicklungsstadien ist in dieser Hinsicht so groß, daß in einigen Fällen (Abb. 1c, 2b) der anfängliche Vorsprung der kernlosen Teile erst nach 22 Tagen von den kernhaltigen Zellen eingeholt wird. Dies ist um so auffälliger, als der Kern die zur Hutbildung nötigen Vorgänge dauernd weiter vorantreibt, wie aus früheren Versuchen bekannt ist und auch bei den hier beschriebenen daran erkannt werden kann, daß der Prozentsatz der von kernlosen Teilen gebildeten Hüte desto höher ist, je weiter

2 K. B et h, Z. Naturforschg. 8 b, 334 [1953]. 